\title{
Introduction: Editors' Introduction to Interacting with Presence
}

\author{
Giuseppe Riva, John Waterworth, and Dianne Murray
}

\section{Why “Interacting with Presence"?}

This volume arose from a longstanding conviction that our subjective experience of information technology is profoundly affected by the extent to which we feel ourselves to be really present in the mediated worlds that the technology makes available to us. 'Presence' is just this "feeling of being inside the mediated world". It is a crucial and increasingly necessary element in both design and usage of many recent and developing interactive technologies. In the same way that 'feeling present', or consciously 'being there', in the physical world around us is based upon perception, physical action and activity in that world, so the feeling of presence in a technologically-mediated environment is a function of the possibilities for interaction.

A more advanced human-centred interaction with systems would provide users with a sense of being there, close to if not equivalent to the experience of actual presence. Creating this sense of presence remains a major challenge and is leading to the development of new interdisciplinary research, combining cognitive psychology, haptic (sense of touch) studies, computer graphics and multimedia design, advanced communication theory and socio-cultural issues. A theory of presence, emerging through this interdisciplinary research, that explores the cognitive and affective roots of sensory perception, is expected to give rise to the design of innovative systems that offer "richer" experiences than any current media and communication technologies.

This, however, is no easy task.

Many researchers, artists and designers have explored the role of the sense of presence in interactive experience. While there is still not a general consensus about what presence actually is, it is fair to say that most investigators agree about what it is not. Presence is not the degree of technological immersion, it is not the same thing as emotional engagement, it is not absorption or attention or action; but all of these have a potential role in understanding the experience of presence in interaction - the experience of interacting with presence. The feeling of presence is a crucial aspect of many recent and developing interactive technologies. The illusion of being present is the key ingredient that gives interactive media the power to affect people profoundly, to change them.

Due to the complexity of the topic, and the interest in this concept, many different attempts to explain the nature of mediated presence have been made. The most basic distinction concerns the issue of technology. For many researchers, mediated presence 
is a direct function of our experience as mediated by specific types of technology. Presence is often defined within this view as the "perceptual illusion of non-mediation" manifested as the disappearance of the medium from the conscious attention of the subject. For others, presence is seen as a broader psychological phenomenon - "inner presence" - related to the control of the subject and to social activity.

Presence is important and is a significant factor in so many different aspects of mediated life because it affects how we behave, what we pay attention to, and how we understand and remember events. The editors believe that, as with emotions, the ability to feeling varying degrees of presence is essential to our survival. Clearly, the experience of presence in a mediated environment is to no small extent a function of the possibilities for interaction, in the same way that feeling present in the physical world is grounded in perception, action and the body. Since these interaction possibilities can be manipulated by design, and in almost unlimited ways, we can conduct experiments in which we measure mediated presence to provide new insights into how and why we feel present in general. The mediated environments we are now creating mix the physical and virtual ever more closely in our experienced everyday lives. To survive and thrive in them, we believe that we will need to consider closely exactly when, to what degree, and in response to which situations we need to feel present - and how we can design our future applications and mediated worlds to achieve that.

"Interacting with Presence" provides an introduction and overview of this increasingly important topic. This timely edited volume presents a range of theoretic perspectives and empirical evidence casting new light on understanding and designing for presence in interaction. Because of its experiential impact on the user, presence is emerging as a key concept for understanding and predicting developments in diverse areas such as interactive entertainment, gaming, psychotherapy, education, scientific visualisation, sports training and rehabilitation, and many more.

With its roots in the development of virtual reality technology, the study of mediated presence has expanded into a range of application disciplines, as discussed in detail in this book. One of the most refreshing and stimulating aspects of presence research is the diversity of views and approaches brought to bear on its explanation and exploration. In tune with this state of affairs, the authors of the chapters in this book explore many different aspects of presence in relation to such topics as the use of tools, social interaction, the nature of mediated embodiment, wellbeing, personal identity and persuasion.

\section{Structure of This Book}

As editors, we have organised the 11 chapters in this volume according to five themes reflected in the sections of the book and outlined below. We have not sought to impose our own views of presence on the other authors. The chapters were chosen initially 
on the basis of the interest and relevance of the proposal to an understanding of presence as mediated through interacting with information technology. The chapters submitted were then reviewed, and most of those were then revised in line with the comments received from two reviewers and the editors. Following this, final versions were submitted and eventually made ready for publication in the book.

The book as a whole comprises a diverse collection of approaches to interacting with presence. What all the chapters share is a conviction that the sense of presence matters. Presence mediated by technology, whether in virtuality, in physical reality, or in some admixture of the two, is important - not merely as a by-product of interaction, but as an outcome with powerful effects.

\section{An Introduction to Presence: Section 1}

Section 1 introduces presence in two chapters that lay out the field as whole, essentially from the perspective of the editors themselves.

Chapter 1, by Giuseppe Riva and Fabrizia Mantovani, presents a general framework for understanding presence in mediated interactions, as 'Extending the Self through the Tools and the Others'. They suggest that presence has a key role in cognition by virtue of having three critical features that cannot be explained by other cognitive processes. Firstly, presence "locates" the Self in an external physical and/or cultural space: the Self is "present" in a space if he/she can act in it. Secondly, presence provides feedback to the Self about the status of its activity: the Self perceives the variations in presence and tunes its activity accordingly. Finally, presence allows the evolution of the Self through the incorporation of tools: tools do not only enable us to extend our reaching space, but may also become part of a plastic neural representation of our body that allows their use without additional cognitive effort.

They extend this analysis by pointing out that social presence enables the subject to identify the Other and to attribute to him or her an ontological status that is different from other objects perceived. Social presence allows interaction and communication through the understanding of the Other's intentions. Ultimately, they point out that social presence permits the evolution of the Self through the identification of "optimal shared experiences" and the incorporation of artefacts - physical and social - linked to them.

Chapter 2, by John and Eva Waterworth, outlines three progressive stages of interactive presence, and the main design challenges raised by each. The first category, termed altered embodiment, refers to the way technology allows us to experience the world with modified or enhanced senses. Echoing the previous chapter, the second category - expanded embodiment - refers to the ways in which technology can push the envelope of the mental body in which one feels present out beyond the physical body. This involves the incorporation of information technology as part of the Self, implying a change in the boundary between the Self and the non-Self, the Other that constitutes 
the world around one. Finally, distributed embodiment refers to how the sense of being present in the world can be separated from that of ownership of a particular body, through the development of new approaches to deploying the technologies of virtual realisation. It is suggested that these three stages of mediated presence are key concepts for understanding possible ways in which designed interactive experiences will affect and continue to change our experience of the world and of our own bodies in the future.

\section{From the Body to the Technology to the Brain: Section 2}

Section 2 provides a view of presence "from the body to the technology to the brain" with two chapters that attempt to ground the phenomena of presence within quite novel theoretical contexts.

We routinely think of mediated presence as a result of the simulation of perceived reality by computer. In Chapter 3, 'Measuring presence in the simulating brain', Daniel Sjölie focuses on how a description of the brain itself as an organ for simulating its environment can inform and illuminate the meaning of brain measurements taken in connection with the sense of presence. Suggested general principles for brain function, such as the free-energy principle, have implications for the ways in which presence can, or cannot, be measured in terms of brain activity. The chapter discusses the implications of accepting the simulating brain concept as a working hypothesis, rather than focusing on arguments for these theories of brain. Such implications are related to how measurements can be connected to some of the most common theoretical descriptions of presence, and to a brief review of previous brain measurement studies that have investigated presence. Overall, the chapter provides a way of interpreting the phenomena of presence, from a new perspective based in the general principles of brain function.

Chapter 4, 'A Framework for Interactivity and Presence in Novel Bodies', by Andrea Stevenson Won and associates, explores the fascinating topic of experiencing mediated presence by interacting with (what are perceived subjectively as) nonhuman bodies. Using trackers, users can control multiple avatar limbs in order to manipulate objects, move through space, and otherwise act in the virtual world. In this chapter the framework developed earlier by two of the authors, Antal Haans and Wijnand A. IJsselsteijn is used to investigate the experience of self-presence in cases of nonhuman avatars or avatars that otherwise differ in ability or control schema from the user's own body, such as avatars with more than two arms. The authors focus on cases where participants inhabit avatars in which the veridical mapping between tracking and rendering is disrupted. 


\section{Experiencing the Others through a Technology: Section 3}

Section 3 examines social presence, the feeling of being engaged with, and connected to others, and the ways it may be mediated by technology.

In Chapter 5, John M. Carroll and colleagues analyse community awareness technologies in terms of their ability to make members feel more present to others and feel that others are more present to them, introducing the concept of "hyperpresence". They focus on place-based technologies and the exciting possibility that they may be used to amplify the sense of presence in the physical world. They draw upon several design investigations of supporting community awareness through the aggregation of RSS feeds and Tweets, digital cultural heritage, volunteer efforts, and fieldwork to understand community awareness designs in a health community.

Chapter 6, by Matthew Hudson and Paul Cairns, looks at the important topic of digital social games and how to measure presence there. Social presence is a key part of the playing experience in such games, but existing measures lack the sophistication to deal with these more diverse gaming situations. The chapter describes the development of a new questionnaire to measure the complex nature of social presence in digital games. The resulting questionnaire consists of 39 items with two main modules addressing competitive and collaborative components of social play.

\section{Using Presence to Increase Wellbeing: Section 4}

Section 4 then focuses on health and how presence can be a factor in efforts to promote improvements in wellbeing.

In Chapter 7, Henry Moller and his co-workers outline a novel paradigm in immersive media technology which is now within reach for clinical application. This seeks to enable an induced state of wellbeing, by creating uplifting states of consciousness rather than generating aversive stimuli or presenting cumbersome tasks for users. Demand for innovative and patient-centred care to alleviate stressrelated and psychosomatic conditions is certainly high in medical settings. A mental health treatment modality that is effective, safe and free of adverse effects is a desirable set of criteria not only from a patient's perspective, but also from the perspective of clinicians who provide healthcare service to patients. In this chapter, positive experiential learning through inspirational/motivational shifts in consciousness through technology is presented as an important route for health promotion in the future.

Sheryl Brahnam (Chapter 8) looks at mediated psychotherapy, and specifically "the uncanny stranger in the room". Taking the perspective of the therapist, this chapter reflects on how, in distance psychotherapy using the telephone and videoconferencing technologies, both the lack of important physical cues and the addition of media artefacts (such as audio/video synchronisation problems and technological glitches) 
can potentially affect the therapeutic relationship and therapeutic presence. The chapter increases our understanding of therapeutic presence and how it is affected when mediated, which will put designers and developers in a better position to appreciate the necessity of supporting and of protecting presence when designing future systems for distance psychotherapy.

Chapter 9, by Villani et al., presents exposure therapy as an effective way to treat anxiety and stress, using Virtual Reality Exposure Therapy, so that the therapeutic experience can be precisely controlled. The authors point out that because of the increased availability of unobtrusive biosensors, and of their integration with other devices, it is possible to measure and take advantage of the sense of presence during the interaction - and so to overcome the limitations of existing protocols for psychological stress and anxiety. They suggest that presence - the experience of "being there" - is influenced by the ability of "making sense there" and by the possibility of learning, by living real experiences in computer mediated environments. They demonstrate that the concept of presence is a critical feature to consider when designing technologymediated protocols, and describe how future investigations will give researchers and clinicians important indications about how to create increasingly effective virtual therapeutic experiences.

\section{Individual Differences in Presence: Section 5}

Finally, Section 5 looks at the relatively neglected topics of individual differences in presence, and of presence as an outcome of persuasive technologies.

In Chapter 10 Anna Felnhofer and Oswald D. Kothgassner confront gender, a personal characteristic that is widely considered to be very important, despite the dearth of substantial findings on the topic. The chapter outlines some of the past research that has been carried out on gender differences in both social and physical presence. Two studies are then described in detail. The first looks at male and female presence experiences in students delivering a speech in front of a virtual audience. The second focuses more broadly on gender differences in young and older adults, while interacting with virtual characters in a collaborative virtual environment. Overall, the results indicate a significant advantage of men over women for physical but not for social presence.

Chapter 11, by Jesse Fox and associates, explores three components of presence (self, social, and spatial presence) and how they relate to persuasion in virtual environments. Presence has often been studied as the mechanism explaining why a virtual environment is successful in persuasion. This chapter points to the importance of understanding the mechanisms that explain presence as an outcome in such persuasive environments. The authors outline an approach whereby virtual environments can be created to cultivate presence and more readily facilitate persuasive goals. 


\section{In Summary}

The wide array of disciplines and applications described in the five Sections strengthens the idea of the importance of presence for real-life applications. As the field continues to grow, we eagerly expect larger on-the-field trials as well as comparisons to existing methods of practice, supporting continued growth of new applications.

Moreover, the book also outlines how the vision of 'optimal presence' can be a strong starting point for giving direction to interactive technology research over the coming five/ten years. Major opportunities to create an optimal presence landscape, based on advanced and intuitive interfaces, can be built in areas such as mobile communications, portable devices, systems integration, embedded computing and intelligent systems design.

Specifically, the book suggests that it is possible to combine the objective of optimal presence with enhancements in information and communication technologies in a move toward a new paradigm: 'Positive Technology'. The main objective of this new paradigm is to use technology to manipulate and enhance features of our personal experience for increasing wellness and generating strength and resilience in individuals, organisations, and society.

In particular it suggests that it is possible to use presence and technology to manipulate the features of our personal experience in three separate but related ways:

1. By structuring personal experience using a goal/meaning, rules and a feedback system;

2. by augmenting personal experience to achieve multimodal and mixed experiences;

3. by replacing personal experience with a synthetic/fictional one.

Nevertheless, the design goal of achieving positive technologies requires an interdisciplinary approach, integrating knowledge and ideas from disciplines such as neuroscience, social and cognitive psychology, multi-sensory perception, cognition, artificial intelligence, multimedia development, video compression or telecoms engineering. In order to build environments which can efficiently transmit remote presence, it will be necessary to incorporate and integrate ongoing insights from these fields into next-generation research for advanced, wideband multi-sensory services and novel telecommunications architectures.

Moreover, the concept of social presence also emphasises the social dimension of innovation, and the ability as well as the willingness of society to use, absorb or adapt to technological opportunities. Alongside technological and economic feasibility, the implications for issues such as social sustainability, privacy, social robustness and fault tolerance may in the longer run determine the success or failure of any presenceenhanced application. 
In the end, we hope that the contents of this book will stimulate more research on technical, cognitive and human factors connected to the sense of "being there" and on how best to use it in communication, education, commerce, design and telemedicine. We thank all the authors for their great work in making this book what we believe it to be - a significant contribution to understanding the roles and importance of presence in a range of interactive situations. We hope our readers enjoy Interacting with Presence! 